\title{
Nanoscale heterogeneous structure of polyacrylonitrile-co-butadiene with different molecular mobilities analyzed by spin-spin relaxation time
}

\author{
Hiroaki Ono ${ }^{1}$, Hirotada Fujiwara ${ }^{2}$ and Shin Nishimura ${ }^{3}$
}

To identify components with different spin-spin relaxation times, $T_{2}$, in the solid-echo pulse proton nuclear magnetic resonance $\left({ }^{1} \mathrm{H}\right.$-NMR) spectra of crude acrylonitrile (AN)-butadiene rubbers (NBRs) with five different AN contents, we tried to understand the inhomogeneity in the crude NBRs in terms of their microstructures and molecular mobilities. The results of small-angle X-ray scattering, differential scanning calorimetry and dynamic mechanical analysis showed that crude NBRs have a singlephase and homogeneous morphology on the nanoscale. The microstructure of the crude NBRs shows alternately copolymerized AN-butadiene (BU) and BU block sequences, as indicated by ${ }^{1} \mathrm{H}-\mathrm{NMR}$ spectra. The $T_{2}$ of the crude NBRs revealed three components with different molecular mobilities, even in homogeneous samples. The content of the highest-mobility component with $T_{2 \mid}$ is negligible. Judging from the AN content dependence of the ${ }^{1} \mathrm{H}$ ratio of these components, the low-mobility component with $T_{2 \mathrm{~s}}$ and high-mobility component with $T_{2 \mathrm{~m}}$ were assigned to the alternately copolymerized AN-BU sequences and BU block sequences, respectively.

Polymer Journal (2013) 45, 1027-1032; doi:10.1038/pj.2013.37; published online 10 April 2013

Keywords: molecular mobility; NBR; pulsed NMR; rubber; spin-spin relaxation time

\section{INTRODUCTION}

Synthetic rubber materials are widely used as sealing materials for various fluids. Rubber materials are designed in terms of the properties of target fluids for seals. For example, in the case of seals for gasoline, polar rubber should be used. In the case of gas seals, the transport of gas through sealing materials should be taken into account. From the perspective of the molecular design of rubbers as sealing materials, the clarification of the relationship between the structure of the rubbers and gas permeation phenomena, the effect of gas on the structure of the rubbers and the physical properties of the rubbers have become increasingly important. The molecular mobility of rubber molecules is one of the most important parameters to consider in designing suitable materials for rubber seals. There are several reports on the molecular mobility of rubber composites evaluated from the spin-spin relaxation time $\left(T_{2}\right)$ of protons $\left({ }^{1} \mathrm{H}\right)$ in rubber molecules determined by free induction decay (FID) signals from pulsed proton nuclear magnetic resonance (pulsed ${ }^{1} \mathrm{H}-\mathrm{NMR}$ ) measurements. ${ }^{1-4}$ Several methods for the evaluation of $T_{2}$ with different pulse sequences are available. The solid-echo pulse sequence method can evaluate relatively short $T_{2}{ }^{5}$. Solid-echo pulse ${ }^{1} \mathrm{H}-\mathrm{NMR}$ is widely used to analyze the crystallinity of polymer materials ${ }^{6}$. Information regarding the inhomogeneous structure of the bulk state of polymers can also be obtained using solid-echo pulse ${ }^{1} \mathrm{H}-\mathrm{NMR}$ as the difference in $T_{2}$ for the components of the inhomogeneous state. Solid-echo pulse ${ }^{1} \mathrm{H}$-NMR analysis can be used to understand the effect of pulsing on the permeation of fluids through sealing rubber materials. The time course of $T_{2}$ can be measured because of the short data acquisition time for FID signals. We have already reported on the solid-echo pulse ${ }^{1} \mathrm{H}-\mathrm{NMR}$ analysis of the time course of $T_{2}$ for vulcanized acrylonitrile (AN)-butadiene rubber (NBR) composites, which are typical rubbers used for hydrogen gas sealing, after highpressure hydrogen exposure to understand the effect of hydrogen on molecular mobility. We identified more than two components with different $T_{2}$ values in crude NBR using solid-echo pulse ${ }^{1} \mathrm{H}$-NMR FID signals, and those $T_{2}$ values and the ${ }^{1} \mathrm{H}$ ratio of each $T_{2}$ value were determined to depend on the AN content of NBR. To understand the transport phenomena of hydrogen through sealing rubber materials according to the mobility of the molecular chains, each component of vulcanized NBR with a distinct $T_{2}$ value should be assigned by taking into account the inhomogeneous structure of crude rubber. Fukumori et al. ${ }^{7}$ reported the solid-echo pulse ${ }^{1} \mathrm{H}-\mathrm{NMR}$ analysis of vulcanized NBR. They found two components with different $T_{2}$ values, a short $T_{2}$ and a long $T_{2}$, which could be assigned to the rubber chains around crosslinks and the chains between crosslinks, respectively.

${ }^{1}$ Department of Hydrogen Energy Systems, Graduate School of Engineering, Kyushu University, Fukuoka, Japan; ${ }^{2}$ The Research Center for Hydrogen Industrial Use and Storage, National Institute of Advanced Industrial Science and Technology, Fukuoka, Japan and ${ }^{3}$ Department of Mechanical Engineering, Faculty of Engineering, Kyushu University, Fukuoka, Japan

Correspondence: Professor S Nishimura, Department of Mechanical Engineering, Faculty of Engineering, Kyushu University, 744 Motooka, Nishi-ku, Fukuoka 819-0395, Japan. E-mail: nishimura.shin.691@m.kyushu-u.ac.jp

Received 30 October 2012; revised 16 January 2013; accepted 20 January 2013; published online 10 April 2013 
However, there are no reports on the concrete assignment of these signals, even for crude rubber materials. NBR is generally polymerized by emulsion polymerization as a random copolymer. Although almost all random copolymers show homogeneous morphology, it has been reported that some random copolymers show an inhomogeneous phase structure. ${ }^{8,9}$ In the case of NBRs, the existence of phaseseparated structures was suggested by the spin-lattice relaxation time $\left(T_{1 \rho}\right)$ analysis of $\mathrm{CH}_{2}$ via the ${ }^{13} \mathrm{C}-\mathrm{NMR}$ inversion recovery method. ${ }^{10}$ According to these reports, the inhomogeneity was caused by an intramolecular inhomogeneous structure composed of segments with different monomer sequences and by large differences in the segmental interaction parameters. However, there are no reports on the relationship between the inhomogeneity in the molecular mobility and in the microstructure of copolymer chains. The inhomogeneity of crude NBRs can be confirmed by small-angle X-ray scattering (SAXS), differential scanning calorimetry (DSC) and dynamic mechanical analysis (DMA). In this study, to identify definitively a wide range of $T_{2}$ components from solid-echo pulse ${ }^{1} \mathrm{H}$-NMR FID signals of crude NBRs, we tried to understand the inhomogeneity in crude NBRs in terms of their microstructures and molecular mobilities.

\section{EXPERIMENTAL PROCEDURE}

Crude NBRs with five different AN contents, low (L), middle (M), middle high $(\mathrm{MH})$, high $(\mathrm{H})$ and very high $(\mathrm{VH})$, were obtained from the Zeon Corporation (Tokyo, Japan): Nipol DN401 (L, AN: 18\%), Nipol 1043 (M, AN: 29\%), Nipol 1042 (MH, AN: 33.5\%), Nipol 1041 (H, AN: 40.5\%) and Nipol DN003(VH, AN: 50\%), respectively. The details of the samples are shown in Table 1. The AN contents described above were specified by the supplier. $M_{\mathrm{e}}$, the molecular weight between entanglements, was estimated from the storage elastic modulus at $30^{\circ} \mathrm{C}$ analyzed by DMA. All measurement samples were used without any further purification.

Solution ${ }^{1} \mathrm{H}-\mathrm{NMR}$ (ECP-400 $400 \mathrm{MHz}$; JEOL Ltd., Akishima, Japan) measurements of the crude NBRs were performed in chloroform-d. The measurement of the crude NBRs was conducted with a single-pulse sequence (pulse width: $23 \mathrm{~ms}$; pulse power: $34 \mathrm{~W}$; acquisition time: $6.87 \mathrm{~ms}$; observation center: 5.0 p.p.m.; sweep width: 20 p.p.m.; scan repetition: 64 times) using a $\phi 5-\mathrm{mm}$ solution probe.

DSC (DSC 204 HP; Netzsch, Selb, Germany) was performed from -100 to $150{ }^{\circ} \mathrm{C}$ in air. The heating rate was $10^{\circ} \mathrm{C} \mathrm{min}-1$.

DMA (itk DVA-225; IT Keisokuseigyo, Osaka, Japan) was performed from -100 to $150^{\circ} \mathrm{C}$. The shear stress frequency was $2.5 \mathrm{~Hz}$. $M_{\mathrm{e}}$ was calculated by using equation (1)

$$
M_{\mathrm{e}}=\frac{\rho R T}{G^{\prime}}
$$

where $\rho$ is the sample density, $R$ is the universal gas constant, $T$ is temperature and $G^{\prime}$ is the storage elastic modulus. $G^{\prime}$ values at $30{ }^{\circ} \mathrm{C}$ were used; thus, $T$ was 303.15 .

SAXS (Nanostar; Bruker AXS, Karlsruhe, Germany) was performed on crude NBR films measuring $5 \mathrm{~mm}$ in thickness. The wavelength of the $\mathrm{Cu} \mathrm{K} \alpha$ $\mathrm{X}$-ray beam was $0.154 \mathrm{~nm}$, and the camera length (sample detector) was

Table 1 Sample information

\begin{tabular}{|c|c|c|c|c|c|c|}
\hline Sample & & Product & $A N(w t \%)$ & $\rho\left(g \mathrm{~cm}^{-3}\right)$ & $M V$ & $\mathrm{M}_{e}\left(\mathrm{~kg} \mathrm{~mol}^{-1}\right)$ \\
\hline $\mathrm{L}$ & & DN401 & 18.0 & 0.94 & 77.5 & 2.0 \\
\hline$M$ & & 1043 & 29.0 & 0.97 & 77.5 & 2.2 \\
\hline $\mathrm{MH}$ & Nipol & 1042 & 33.5 & 0.98 & 77.5 & 1.8 \\
\hline V & & 1041 & 40.5 & 1.00 & 82.5 & 2.5 \\
\hline VH & & DN003 & 50.0 & 1.02 & 77.5 & 2.2 \\
\hline
\end{tabular}

Abbreviations: AN, official value of acrylonitrile content of crude NBRs; $\rho$, official value of density of crude NBRs; MV, official value of Mooney viscosity; $M_{\mathrm{e}}$, molecular weight between entanglements from storage elastic modulus at $30^{\circ} \mathrm{C}$ analyzed by dynamic mechanical analysis; NBR, acrylonitrile-butadiene rubber.
$1053 \mathrm{~mm}$. Scattering patterns were collected with a two-dimensional detector (Vantec-Hi Star; Bruker AXS).

The solid-echo $\left(90^{\circ}{ }_{x}-\tau-90^{\circ}\right)$ FID signals of the samples were analyzed by using pulsed ${ }^{1} \mathrm{H}-\mathrm{NMR}$ (minispec mq20, $20 \mathrm{MHz}$; Bruker BioSpin, Karlsruhe, Germany) at $30^{\circ} \mathrm{C}$. Samples $(\phi 10 \mathrm{~mm} \times 10 \mathrm{~mm})$ were placed in $\phi 10 \mathrm{~mm}$ Pyrex tubes. The $T_{2}$ values and ${ }^{1} \mathrm{H}$ ratios of the $T_{2}$ values were calculated from the obtained FID signals by the converged equation (2) using the least-squares method,

$$
\text { Int }=\sum_{i=1}^{N} A_{i} \exp \left(-\left(\frac{1}{\alpha_{i}}\right)\left(\frac{t}{T_{2 i}}\right)^{\alpha_{i}}\right)+c
$$

where $A_{i}$ is the intensity at time $0, t$ is time, $T_{2 i}$ is the $T_{2}$ value of $i$ th component and $\alpha_{i}$ is the FID signal shape constant. The ${ }^{1} \mathrm{H}$ ratios of $T_{2 i}$ were evaluated using $M_{i}$, as shown in equation (3):

$$
M_{i}=A_{i} / \sum_{i} A_{i}
$$

\section{RESULTS AND DISCUSSION}

\section{Monomer sequence distribution of crude NBRs}

To determine the monomer sequence distribution of the crude NBRs, the solution ${ }^{1} \mathrm{H}-\mathrm{NMR}$ spectra of these samples were measured. Figure 1 shows the spectrum of $\mathrm{MH}$. The protons of methine $(\mathrm{CH})$ at the center of the triad were observed in the regions of 2.5-2.9 and 5.2-5.7 p.p.m. The signals of $\mathrm{CH}$ were divided into five regions: $5.5-5.67,2.85,2.58,2.85$ and 5.2-5.43 p.p.m., which were assigned to the triads of NBR, ABA, BAA, BAB, BBA and BBB, respectively, ${ }^{11}$ where $\mathrm{A}$ is an $\mathrm{AN}$ unit and $\mathrm{B}$ is a butadiene $(\mathrm{BU})$ unit. The $\mathrm{CH}$ of AAA, which were observed at 3.1 p.p.m. ${ }^{12}$ in polyacrylonitrile, were not detected in the spectra of the crude NBRs. The average contents of $\mathrm{AN}, \mathrm{BU}$ and triad distributions were calculated from the area of these signals.

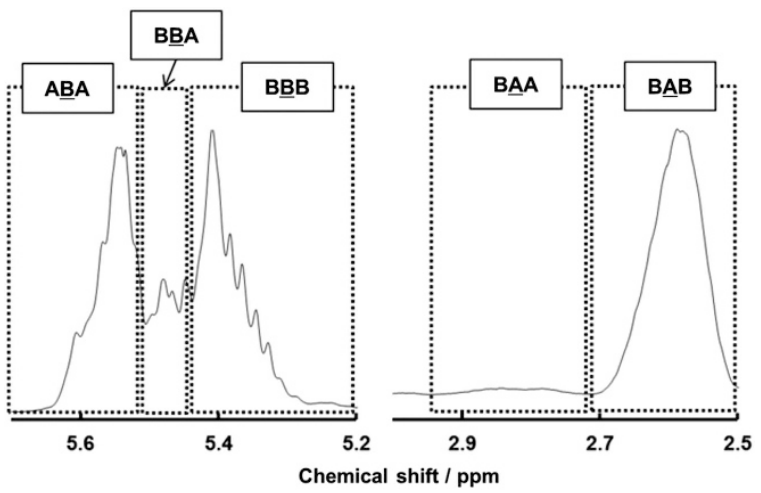

Figure 1 Proton nuclear magnetic resonance $\left({ }^{1} \mathrm{H}-\mathrm{NMR}\right)$ spectrum of crude acrylonitrile-butadiene rubber (NBR) (MH) in chloroform-d.

\begin{tabular}{|c|c|c|c|c|c|c|c|c|}
\hline \multirow[b]{2}{*}{ Samples } & \multicolumn{8}{|c|}{ Morlar ratio (\%) } \\
\hline & $A N$ & $B U$ & $A A A$ & $B A B$ & $B A A$ & $B B B$ & $B B A$ & $A B A$ \\
\hline L & 21 & 79 & 0 & 21 & 0 & 48 & 13 & 19 \\
\hline M & 30 & 70 & 0 & 30 & 0 & 29 & 13 & 28 \\
\hline $\mathrm{MH}$ & 35 & 65 & 0 & 34 & 1 & 28 & 12 & 26 \\
\hline $\mathrm{H}$ & 41 & 59 & 0 & 39 & 2 & 16 & 10 & 33 \\
\hline $\mathrm{VH}$ & 49 & 51 & 0 & 38 & 10 & 5 & 5 & 42 \\
\hline
\end{tabular}

Table 2 Average chemical composition and triad distributions of crude NBR 
The average chemical composition and triad distribution of the crude NBRs determined by solution ${ }^{1} \mathrm{H}-\mathrm{NMR}$ are shown in Table 2.

The contents of $\mathrm{ABA}$ and $\mathrm{BAB}$ increased with the $\mathrm{AN}$ content. The contents of BBA and BBB clearly decreased with increasing AN content. These results suggest that in crude NBRs, AN units prefer alternate or random copolymerization with $\mathrm{BU}$ units; however, BU units prefer the formation of BU blocks.

The average sequence lengths of $\mathrm{AN}\left(L_{n}[\mathrm{AN}]\right)$ and $\mathrm{BU}\left(L_{n}[\mathrm{BU}]\right)$ in each crude NBR were calculated using equations (4) and (5), ${ }^{13}$

$$
\begin{aligned}
& L_{n}[\mathrm{AN}]=\left(F_{\mathrm{BAB}}+F_{\mathrm{BAA}}+F_{\mathrm{AAA}}\right) /\left(F_{\mathrm{BAB}}+\frac{F_{\mathrm{BAA}}}{2}\right) \\
& L_{n}[\mathrm{BU}]=\left(F_{\mathrm{ABA}}+F_{\mathrm{BBA}}+F_{\mathrm{BBB}}\right) /\left(F_{\mathrm{ABA}}+\frac{F_{\mathrm{BBA}}}{2}\right)
\end{aligned}
$$

where $F_{\mathrm{AAA}}, F_{\mathrm{BAB}}, F_{\mathrm{BAA}}, F_{\mathrm{BBB}}, F_{\mathrm{BBA}}$ and $F_{\mathrm{ABA}}$ are the molar fractions of the triads. The relationship between the $\mathrm{AN}$ content and $L_{n}[\mathrm{AN}]$, $L_{n}[\mathrm{BU}]$ is illustrated in Figure 2. As shown in Figure 2, $L_{n}[\mathrm{BU}]$ decreased proportionally with the increase in the AN content. In contrast, $L_{n}[\mathrm{AN}]$ remained constant. The relationship between the mole fraction of $\mathrm{BU}$ units in the polymer $\left(F_{\mathrm{BU}}\right)$ and the mole fraction of feedstock AN monomer $\left(f_{\mathrm{AN}}\right)$ was calculated using equation $(6),{ }^{14}$

$$
F_{\mathrm{BU}}=\frac{r_{\mathrm{BU}} f_{\mathrm{BU}^{2}}+f_{\mathrm{BU}} f_{\mathrm{AN}}}{r_{\mathrm{BU}} f_{\mathrm{BU}}^{2}+2+r_{\mathrm{AN}} f_{\mathrm{AN}^{2}}}
$$

where $f_{\mathrm{AN}}$ and $f_{\mathrm{BU}}$ are the mole fractions of feedstock $\mathrm{AN}$ and $\mathrm{BU}$ monomers, respectively, and $r_{\mathrm{AN}}(=0.03)$ and $r_{\mathrm{BU}}(=0.2)$ are the $\mathrm{AN}$

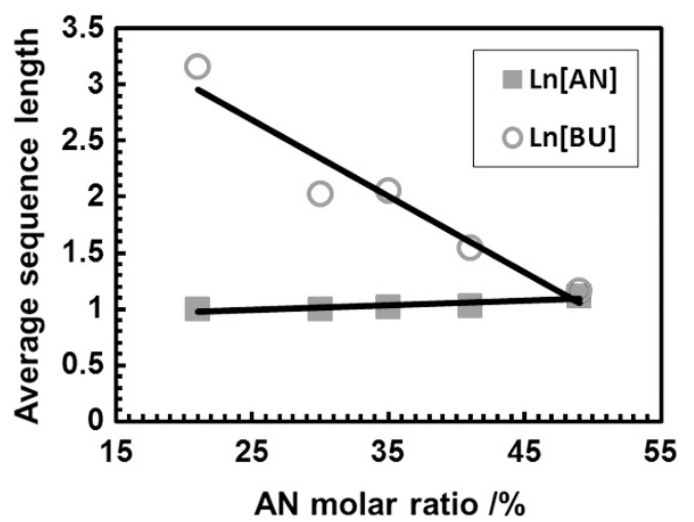

Figure 2 Relationship between acrylonitrile (AN) content and average sequence length of $A N\left(L_{n}[A N]\right)$ and butadiene $(B U)\left(L_{n}[B U]\right)$. A full color version of this figure is available at Polymer Journal online.

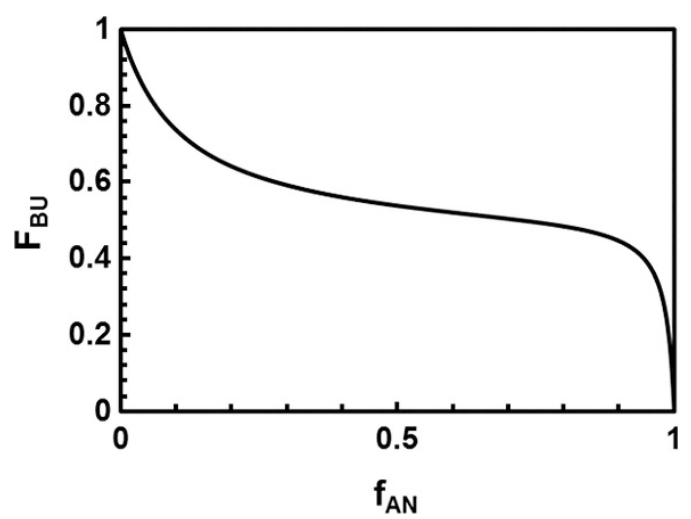

Figure 3 Relationship between molar fraction of butadiene (BU) in polymer chains and molar fraction of feedstock acrylonitrile (AN) monomer. and BU monomer reactivity ratios, respectively. ${ }^{15}$ The calculated results are shown in Figure 3. According to this figure, in the region of $f_{\mathrm{AN}}=0.2-0.8, \quad f_{\mathrm{BU}}$ was nearly 0.5 . During the early stages of copolymerization, an AN-BU alternate copolymer was produced, and during the later stages, $\mathrm{BU}$ blocks were produced by the remaining $\mathrm{BU}$ monomer.

According to these results, crude NBR chains consist of two components, a BU block sequence and an alternately copolymerized $\mathrm{AN}-\mathrm{BU}$ sequence, at low and high AN contents components, respectively.

\section{Morphological analysis of crude NBRs by SAXS}

We tried to estimate the size of aggregated AN-BU chains by using SAXS, and the obtained scattering profile was analyzed by Fankuchen's method. ${ }^{16}$ For monodisperse aggregates with a radius of gyration $R$, the SAXS intensity can be described by Guinier's method using equation (7),

$$
I(q)=I(0) \exp \left(-\frac{q^{2} R_{\mathrm{g}}^{2}}{3}\right)
$$

where $I(q)$ is the SAXS intensity, $I(0)$ is the intensity of $q=0, q$ is the scattering vector and $R_{\mathrm{g}}$ is the radius of gyration. However, for polydisperse aggregates with radii of gyration $R_{\mathrm{g}}$, the distribution of $R_{\mathrm{g}}$ can be calculated by Fankuchen's method using equation (8):

$$
I(q)=\sum_{i} I(0)_{i} \exp \left(-\frac{q^{2} R_{\mathrm{g} i}^{2}}{3}\right)
$$

Equation (8) is a summation of equation (7). Thus, equation (8) can be described by a double logarithm as follows:

$$
\ln \mathrm{I}(\mathrm{q})=\sum_{i} \ln I(0){ }_{i} \exp \left(-\frac{q^{2} R_{\mathrm{gi}}^{2}}{3}\right)
$$

Thus, according to equation (7), $R_{\mathrm{g} i}$ can be calculated from the slope of the plot of $\ln (I(q))$ vs $q^{2}$, the so-called Guinier plot. The relationship between the slope $\left(S_{i}\right)$ and $R_{\mathrm{g} i}$ and the relationship between $R_{\mathrm{g} i}$ and the particle radius $\left(R_{i}\right)$ are described by equations (10) and (11), respectively. The mass ratio of components with $R_{\mathrm{g} i}$ can be calculated by equation (12): ${ }^{17}$

$$
\begin{aligned}
& S_{i}=-\frac{R_{\mathrm{g} i}^{2}}{3} \\
& R_{i}=\sqrt{\frac{5}{3}} R_{\mathrm{g} i} \\
& W\left(R_{\mathrm{g} i}\right)=\frac{I(0)_{i}}{R_{\mathrm{g} i}^{3}} / \sum_{i} \frac{I(0)_{i}}{R_{\mathrm{g} i}^{3}}
\end{aligned}
$$

Guinier plots of the crude NBRs are shown in Figure 4, and sample slopes of the Guinier plots are shown in Figure 5. The estimated values of $R_{i}$ and $W\left(R_{i}\right)$ are shown in Table 3 . Three slopes were obtained from the Guinier plots. As shown in Table 3, the obtained $R_{i}$ were $0.13-0.37,3.62-5.21$ and $11.7-14.6 \mathrm{~nm}$. The two larger domains exhibited quite small mass ratios. According to the results of SAXS, the crude NBRs were regarded as having a single-domain morphology, that is, nanodomain aggregates with $R_{i}=0.13-0.37 \mathrm{~nm}$. In other words, the crude NBRs exhibited a homogeneous morphology on the nanoscale.

Thermal analysis for glass transition temperature

From the results of morphological analysis, it was concluded that the crude NBRs were single phase. To confirm the uniformity of the 
materials, DSC and DMA measurements were performed on crude NBR samples to evaluate their glass transition temperatures $\left(T_{\mathrm{g}}\right)$.

Figure 6 shows the DSC curves of the crude NBRs. The curves show two endothermic peaks for all samples. The $T_{\mathrm{g}}$ of each sample was determined as the downward peak of the first-order derivative of the corresponding DSC curve. The main endothermic peak temperatures below $0{ }^{\circ} \mathrm{C}$ were designated as the $T_{\mathrm{g}}$ values of the crude NBRs.

Chandler et al. ${ }^{18}$ reported that the results of differential thermal analyses for crude NBRs with $<36 \%$ AN showed two $T_{\mathrm{g}}$ values below $0{ }^{\circ} \mathrm{C}$ because of the existence of a phase-separated structure in the samples. ${ }^{18}$ In the case of emulsion-polymerized NBR, the monomer sequence distribution was dominated by the polymerization temperature and conversion rate. ${ }^{19}$ However, our DSC results for crude NBRs show only one $T_{\mathrm{g}}$.

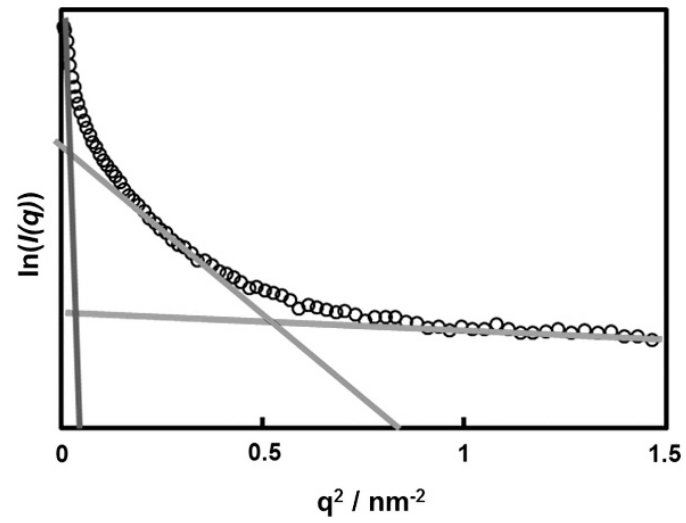

Figure 4 Guinier plot and $\mathrm{MH}$ slope. A full color version of this figure is available at Polymer Journal online.

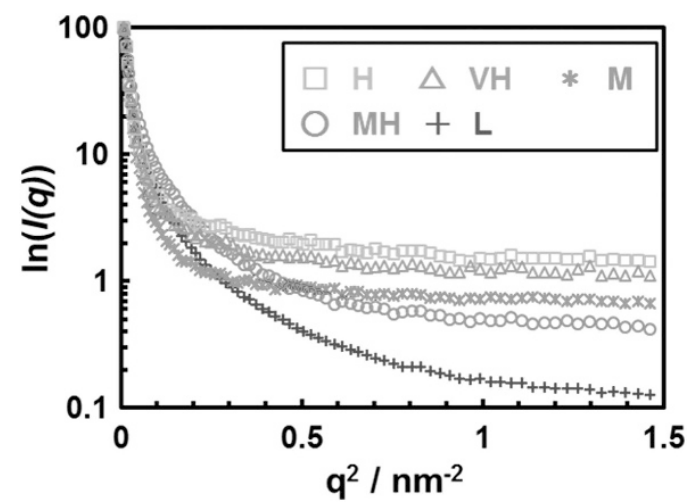

Figure 5 Guinier plot of crude acrylonitrile-butadiene rubbers (NBRs). A full color version of this figure is available at Polymer Journal online.
Figure 7 shows the temperature dependence of $\tan \delta$ measured by DMA for crude NBR samples. $T_{\mathrm{g}}$ was determined as the peak temperature of $\tan \delta$. The $\tan \delta$ values showed a single peak in all samples. These peak temperatures were designated the $T_{\mathrm{g}}$ values. $T_{\mathrm{g}}$ increased as the AN content increased. Thus, only one $T_{\mathrm{g}}$ was observed in not only the DSC profiles but also the DMA profiles.

Judging from our SAXS, DSC and DMA results, the crude NBRs used in this study showed a single-phase and homogeneous morphology.

\section{Pulsed ${ }^{1} \mathrm{H}-\mathrm{NMR}$ analysis of crude NBRs}

The observed FID signals and fitted FIDs for crude NBRs at $30{ }^{\circ} \mathrm{C}$ are shown in Figure 8 . The FID signals obtained were fitted by

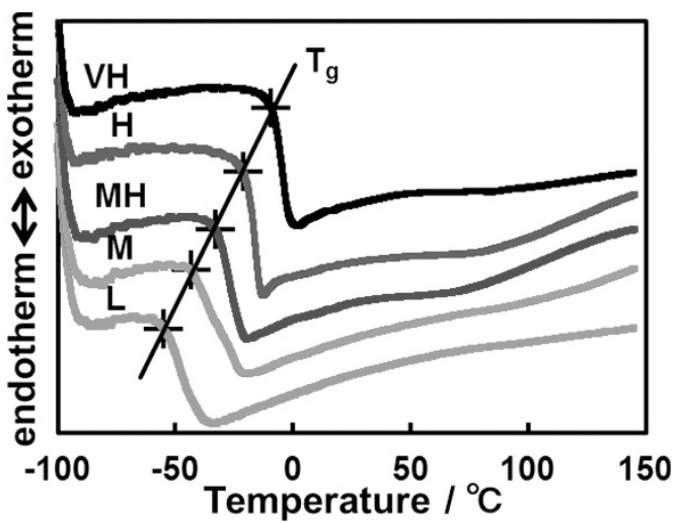

Figure 6 Differential scanning calorimetry (DSC) curves of crude acrylonitrile-butadiene rubbers (NBRs). A full color version of this figure is available at Polymer Journal online.

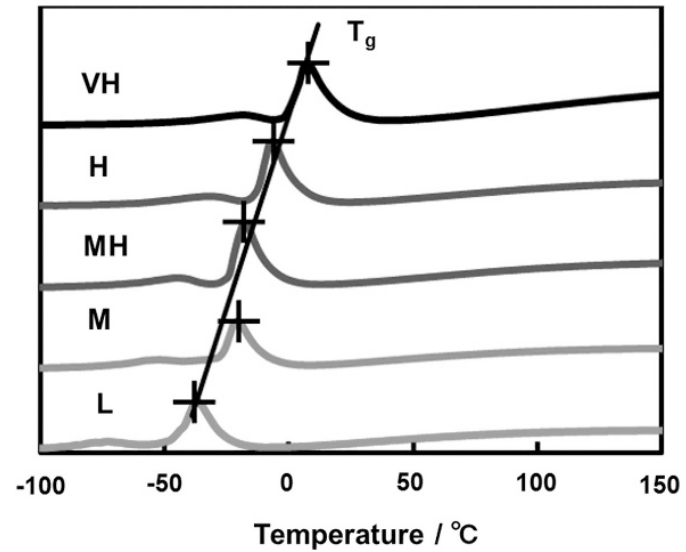

Figure $7 \operatorname{Tan} \delta$ of crude acrylonitrile-butadiene rubbers (NBRs). A full color version of this figure is available at Polymer Journal online.

Table 3 Radius of domain $\left(R_{i}\right)$ and weight fraction of domain with $R_{i}$ of crude NBRs

\begin{tabular}{|c|c|c|c|c|c|c|c|c|c|}
\hline \multicolumn{2}{|c|}{$L$} & \multicolumn{2}{|c|}{$M$} & \multicolumn{2}{|c|}{$M H$} & \multicolumn{2}{|c|}{$H$} & \multicolumn{2}{|c|}{$V H$} \\
\hline $\mathrm{R}_{\mathrm{i}}(n m)$ & $W\left(R_{i}\right)$ & $\mathrm{R}_{\mathrm{i}}(n m)$ & $W\left(R_{i}\right)$ & $\mathrm{R}_{\mathrm{i}}(n m)$ & $W\left(R_{i}\right)$ & $\mathrm{R}_{\mathrm{i}}(n m)$ & $W\left(R_{\mathrm{i}}\right)$ & $\mathrm{R}_{\mathrm{i}}(n m)$ & $W\left(R_{i}\right)$ \\
\hline 0.37 & 1.00 & 0.23 & 1.00 & 0.26 & 1.00 & 0.13 & 1.00 & 0.16 & 1.00 \\
\hline 3.84 & $4.69 \times 10^{-4}$ & 5.21 & $1.34 \times 10^{-5}$ & 4.46 & $8.86 \times 10^{-5}$ & 3.62 & $4.86 \times 10^{-6}$ & 4.40 & $6.41 \times 10^{-6}$ \\
\hline 11.8 & $1.09 \times 10^{-5}$ & 11.82 & $3.17 \times 10^{-6}$ & 14.6 & $1.48 \times 10^{-6}$ & 11.9 & $4.95 \times 10^{-7}$ & 11.7 & $1.03 \times 10^{-6}$ \\
\hline
\end{tabular}

Abbreviations: NBR, acrylonitrile butadiene rubber; $R_{i}$, radius of domain; $W\left(R_{i}\right)$, weight fraction of domain with $R_{i}$. 


\begin{tabular}{l} 
- Experimental data \\
- Fitted FID \\
$-\mathrm{T}_{2 \mathrm{~s}}$ \\
$--\mathrm{T}_{2 \mathrm{~m}}$ \\
$\ldots \ldots . . \mathrm{T}_{21}$ \\
\hline
\end{tabular}
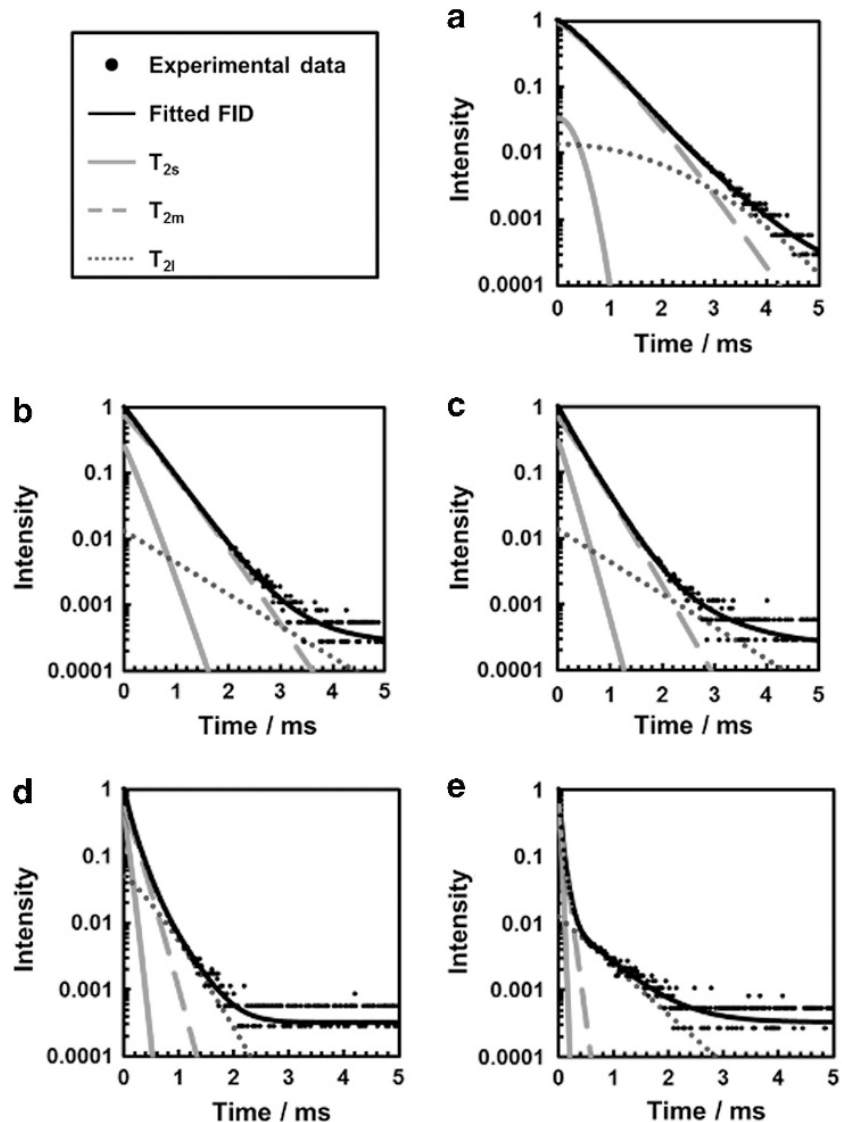

Figure 8 Free induction decay (FID) signals of pulsed proton nuclear magnetic resonance $\left({ }^{1} \mathrm{H}-\mathrm{NMR}\right)$ spectra with fitting results for crude (NBRs): (a) $\mathrm{L}$, (b) $\mathrm{M}$, (c) $\mathrm{MH}$, (d) $\mathrm{H}$ and (e) $\mathrm{VH}$. A full color version of this figure is available at Polymer Journal online.

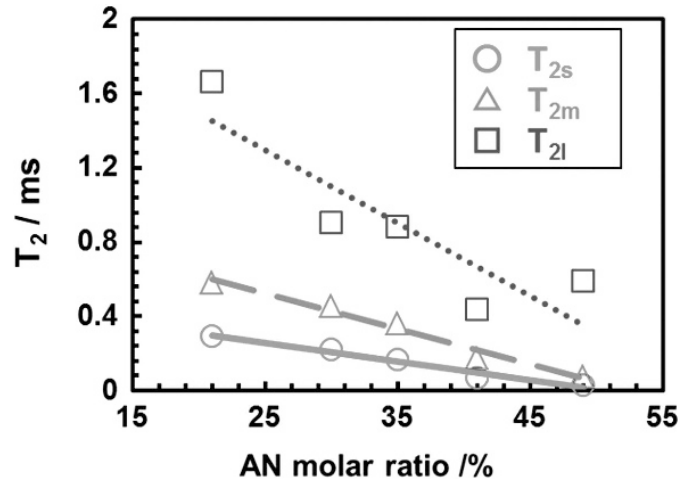

Figure 9 The relationship between acrylonitrile (AN) content and $T_{2}$. A full color version of this figure is available at Polymer Journal online.

equation (1) using the least-squares method. $A_{i}, N, \alpha_{i}$ and $T_{2 i}$ in equation (1) were treated as fitting parameters. As indicated by the fitting results, the number of components $N$ for each crude NBR was 3. The residual sums of the squares of the fitting results were below $10^{-4}$. The FID signals of all of the samples could be separated into three $T_{2}$ components denoted $T_{2 \mathrm{~s}}, T_{2 \mathrm{~m}}$ and $T_{21}$. The relationship between AN content and $T_{2}$ analyzed by pulsed ${ }^{1} \mathrm{H}-\mathrm{NMR}$ is illustrated in Figure 9. $T_{2 \mathrm{~s}}, T_{2 \mathrm{~m}}$ and $T_{21}$ decreased proportionally with increasing AN content. Figure 10 shows the amount of each component as the ${ }^{1} \mathrm{H}$ ratio. As shown in Figure 10, the ${ }^{1} \mathrm{H}$ ratio of the component with

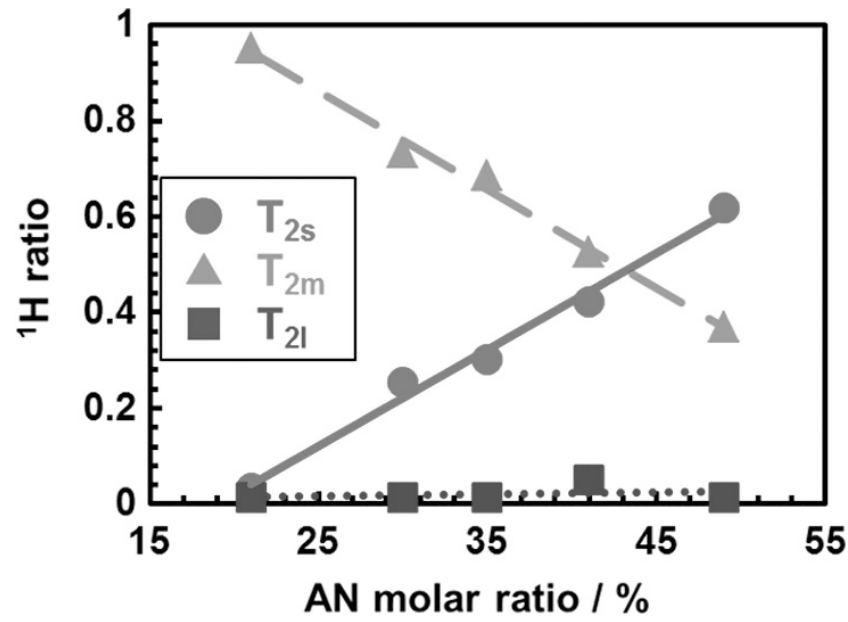

Figure 10 The relationship between acrylonitrile (AN) content and ${ }^{1} \mathrm{H}$ ratio with $T_{2}$. A full color version of this figure is available at Polymer Journal online.

$T_{2 \mathrm{~s}}$ increased and that with $T_{2 \mathrm{~m}}$ decreased as the AN content increased. The ${ }^{1} \mathrm{H}$ ratio with $T_{21}$ was quite small and remained constant. These results indicate that there were two components with different molecular mobilities in the crude NBRs. The mobility of each component depends on the AN content of the component. The number of polar monomer units increases with AN content. The increase in the number of polar monomer units causes a decrease in molecular mobility. Kwak and $\mathrm{Kim}^{10}$ reported the observation of local phase separation into sea-island structures in crude NBRs from the analysis of the spin-lattice relaxation time $T_{1 \rho}$ of $\mathrm{CH}_{2}$. Faghihi et $a .^{8}$ reported that random copolymers of butyl acrylate and methyl methacrylate showed nanophase segregation according to DMA, DSC and ${ }^{13} \mathrm{C}$-NMR spin diffusion experiments. Kipper et al. ${ }^{9}$ reported that random copolymers of 1,6-bis( $p$-carboxyphenoxy)hexane and sebacic acid undergo microphase separation because of large differences in the segment-segment interaction parameters. In this study, the crude NBRs did not show a phase separation structure. Two components with different molecular mobilities were observed in the crude NBRs.

Assignment of components with $T_{2 \mathrm{~s}}$ and $T_{2 \mathrm{~m}}$ of crude NBRs According to the results of solution ${ }^{1} \mathrm{H}-\mathrm{NMR}$, crude NBR chains consist of two components, a BU block sequence and an alternately copolymerized AN-BU sequence, at low and high AN contents, respectively. According to the results of SAXS, the crude NBRs showed a single-domain morphology, that is, the aggregation of nanodomains. The DSC curves and $\tan \delta$ peaks of the crude NBRs show one $T_{\mathrm{g}}$. The crude NBRs were determined to exhibit homogeneous morphology on the nanoscale. The spin-spin relaxation time, $T_{2}$, determined by pulsed ${ }^{1} \mathrm{H}-\mathrm{NMR}$ for crude NBRs showed mainly two components with different molecular mobilities, even in homogeneous samples. The ${ }^{1} \mathrm{H}$ ratio of $T_{2 \mathrm{~s}}$ increased and that of $T_{2 \mathrm{~m}}$ decreased with increasing AN content. As shown in Table 1, the molecular weights between entanglements, $M_{\mathrm{e}}$, for all samples were all close to $2.0 \mathrm{~kg} \mathrm{~mol}^{-1}$, despite the differences in the AN content among the samples. Judging from the dependence of the ${ }^{1} \mathrm{H}$ ratio on the AN content, $T_{2 s}$ and $T_{2 \mathrm{~m}}$ were assigned to the alternately copolymerized $\mathrm{AN}-\mathrm{BU}$ sequence, which is strongly constrained by intermolecular interactions between polar nitrile groups and BU block sequences, which are weakly constrained. 


\section{CONCLUSIONS}

To understand the transport phenomena of hydrogen through sealing rubber materials by examining the mobility of their molecular chains, each component with a distinct $T_{2}$ in vulcanized NBR should be assigned by taking into account the inhomogeneous structure of crude rubber. In this study, to identify definitively a wide range of $T_{2}$ components from the solid-echo pulsed ${ }^{1} \mathrm{H}-\mathrm{NMR}$ FID signals of crude NBRs, we tried to understand the inhomogeneity of the crude NBRs in terms of their monomer sequence distribution and molecular mobility. The crude NBRs show homogeneous morphology on the nanoscale; however, the spin-spin relaxation time, $T_{2}$, determined by pulsed ${ }^{1} \mathrm{H}-\mathrm{NMR}$ for the crude NBRs showed three components with different molecular mobilities, even in homogeneous samples. The content of the highest-mobility component with $T_{21}$ was negligibly small. Judging from the dependence of the ${ }^{1} \mathrm{H}$ ratio on the AN content in these components, the low-mobility component with $T_{2 s}$ and the high-mobility component with $T_{2 \mathrm{~m}}$ were assigned to the alternately copolymerized AN-BU sequence and BU block sequence, respectively. The inhomogeneity of crude NBR may be attributed to its monomer sequence distribution.

\section{ACKNOWLEDGEMENTS}

This research has been supported by the New Energy and Industrial Technology Development Organization (NEDO) 'Fundamental Research Project on Advanced Hydrogen Science (2006-2012)'.

1 Borgia, G. C., Fantazzini, P., Ferrando, A. \& Maddinelli, G. Characterisation of crosslinked elastomeric materials by ${ }^{1} \mathrm{H}$ NMR relaxation time distributions. Magn. Reson. Imaging 19, 405-409 (2001).

2 Whittaker, A. K., Bremner, T. \& Zelaya, F. O. The effect of field inhomogeneities and molecular diffusion on the NMR transverse relaxation behaviour of polymer melts. Polymer 36, 2159-2164 (1995).
3 Garrido, L., Mark, J. E., Sun, C. C., Ackerman, J. L. \& Chang, C. NMR characterization of elastomers reinforced with in situ precipitated silica. Macromolecules 24 4067-4072 (1991).

4 O'Brien, J., Cashell, E., Wardell, G. E. \& McBrierty, V. J. An NMR investigation of the interaction between carbon black and cis-polybutadiene. Macromolecules 9, 653-660 (1976).

5 Fukumori, K., Kurauchi, T. \& Kamigaito, O. Pulsed NMR study of elastomeric block copolymer under deformation. J. Appl. Polym. Sci. 38, 1313-1334 (1989).

6 Uehara, H., Yamanobe, T. \& Komoto, T. Relationship between solid-state molecular motion and morphology for ultrahigh molecular weight polyethylene crystallized under different conditions. Macromolecules 33, 4861-4870 (2000).

7 Fukumori, K., Norio Sato, N. \& Kurauchi, T. Deformation behaviors of nitrile rubber vulcanizates as studied by pulsed NMR: effects of carbon black fillers. Nippon Gomu Kyoukaishi 61, 561-566 (1988).

8 Faghihi, F., Mohammadi, N. \& Hazendonk, P. Effect of restricted phase segregation and resultant nanostructural heterogeneity on glass transition of nonuniform acrylic random copolymers. Macromolecules 44, 2154-2160 (2011).

9 Kipper, M. J., Hou, S. -S., Seifert, S., Thiyagarajan, P., Schmidt-Rohr, K. \& Narasimhan, B. Nanoscale morphology of polyanhydride copolymers. Macromolecules 38, 8468-8472 (2005).

10 Kwak, S. -Y. \& Kim, S. Y. Microphase structures of polymers containing structural heterogeneity as probed by n.m.r. spin-lattice relaxation analysis. Polymer 39, 4099-4105 (1998).

11 Anachkov, M. P., Stefanova, R. V. \& Rakovsky, S. K. ${ }^{1}$ H NMR study of monomer sequence distributions in some commercial acrylonitrile-butadiene copolymers. $\mathrm{Br}$. Polym. J. 21, 429-432 (1989).

12 Pai Verneker, V. R. \& Shaha, B. On coloration of polyacrylonitrile: a nuclear magnetic resonance study. Macromolecules 19, 1851-1856 (1986).

13 Matsuda, H., Asakura, T. \& Miki, T. Triad sequence analysis of poly(ethylene/ butylene terephthalate) copolymer using ${ }^{1} \mathrm{H}$ NMR. Macromolecules $35,4664-4668$ (2002)

14 Hiemenz, P.C \& Lodge, T. P. Polymer Chemistry. 2nd edn. Vol. 168 (CRC Press, New York, NY, USA, 2007)

15 Greenley, R.Z. in Polymer Handbook. 4th edn (eds. Brandrup, J., Immergut, E. H., Grulke, E. A., Abe, A. \& Bloch, D. R.) Ch. 2, 203 (Wiley, New York, NY, USA, 1999)

16 Wang, X. -S., Kim, H. -K., Fujita, Y., Sudo, A., Nishida, H. \& Endo, T. Relaxation and reinforcing effects of polyrotaxane in an epoxy resin matrix. Macromolecules 39, 1046-1052 (2006).

17 Rigaku X-ray Research Laboratory X-ray diffraction Handbook 115-116 (Rigaku, Japan, 2000)

18 Chandler, L. A. \& Collins, E. A. Multiple glass transitions in butadiene-acrylonitrile copolymers. J. Appl. Polym. Sci. 13, 1585-1593 (1969).

19 Hashimoto, K. in Rubber Industrial Handbook (The Society of Rubber Science and Technology Ch. 2, 238The Society of Rubber Science and Technology, 1994). 